\title{
Desafios à atuação dos trabalhadores de Vigilância Sanitária nos serviços de saúde
}

\section{Challenges to the performance of health surveillance workers in health services}

\author{
Ediná Alves Costa' \\ Eliana Auxiliadora \\ Magalhães Costa" iD \\ Mariluce Karla Bomfim \\ de Souza' (iD
}

Patrícia Sodré Araújo"

Gisélia Santana Souza'

Yara Oyram Ramos Lima ${ }^{1, *}$

Bianca Maria Santos da Paz' (iD)

I Instituto de Saúde Coletiva, Universidade Federal da Bahia, Salvador, BA, Brasil

" Universidade do Estado da Bahia, Salvador, BA, Brasil

\section{* E-mail: oyram@ufba.br}

Recebido: 13 dez 2020

Aprovado: 12 nov 2021

\section{RESUMO}

Introdução: A vigilância sanitária é uma função precípua do Estado para proteção da saúde individual e coletiva mediante ações de controle de riscos, atuais ou potenciais, presentes na produção de bens e serviços, e integra o Sistema Único de Saúde. A vigilância sanitária de serviços de saúde é parte do escopo de atuação da Vigilância Sanitária (Visa) para o controle dos riscos e iatrogenias nos serviços de saúde. Objetivo: Analisar desafios à atuação dos trabalhadores de Visa nos serviços de saúde. Método: 0 estudo, de abordagem qualitativa, integra uma pesquisa financiada pelo CNPQ para análise do controle sanitário realizado pelas Visa em serviços de saúde da atenção básica e especializada. Utilizou-se a técnica de Grupo Focal com trabalhadores da Visa de oito estados brasileiros. As falas foram transcritas e analisadas segundo a técnica de análise de conteúdo temática, sistematizadas em duas categorias: Gestão e Organização dos Serviços e Condições de Trabalho. Resultados: Identificou-se problemas e desafios, tais como: incipiente planejamento das ações; dificuldades na organização do trabalho decorrentes da rotatividade de gestores, da falta de recursos humanos e tecnológicos, da ausência de coordenação e da integração dos níveis do Sistema Nacional de Vigilância Sanitária. Emergiram insatisfações e desafios sobre condições de trabalho, precarizações dos vínculos, baixos salários, equipes incompletas e necessidade de concursos públicos e de valorização do trabalho. Conclusões: A atuação da Visa em serviços de saúde requer a superação de múltiplos desafios, que perpassam a integração das ações nos distintos níveis de gestão e questões de capacitação, incorporação de tecnologias e de readequação de processos de trabalho.

PALAVRAS-CHAVE: Vigilância Sanitária; Trabalhador da Saúde; Gestão de Serviços de Saúde; Saúde Coletiva

\section{ABSTRACT}

Introduction: Health surveillance (Visa) is a primary function of the State for the protection of individual and collective health through risk control actions, current or potential, present in the production of goods and services, and is part of SUS (Brazilian Universal Health System). Health surveillance of health services is part of Visa's scope of action for the control of risks and iatrogenic diseases in health services. Objective: To analyze challenges to the performance of Visa in health services. Method: The study, with a qualitative approach, integrates research funded by CNPq to analyze the sanitary control carried out by Visa in health services of primary and specialized care; the Focus Group technique was used with Visa workers from 8 Brazilian states. The speeches were transcribed and analyzed according to the thematic content analysis technique, systematized in two categories: Management and Organization of Services, and Working Conditions. Results: Problems and challenges were identified, such as: incipient planning of actions; difficulties in the organization of work, resulting from the turnover of managers, the lack of human and technological resources, and the lack of coordination and integration of the levels of the National Health Surveillance System. Dissatisfactions 
and challenges about working conditions, precarious employment conditions, low wages, incomplete teams and the need for public tenders and work valorization emerged. Conclusions: Visa's performance in health services requires overcoming multiple challenges, which permeate the integration of actions at different levels of management and training issues, incorporation of technologies and readjustment of work processes.

KEYWORDS: Health Surveillance; Health Worker; Health Services Management; Collective Health

\section{INTRODUÇÃO}

A vigilância sanitária constitui uma das áreas mais complexas da saúde coletiva, integra o Sistema Único de Saúde (SUS) e responde pela regulação sanitária, proteção da saúde, prevenção e controle de riscos relacionados a produtos, tecnologias, processos e serviços de interesse da saúde. Devido à função regulatória, as ações de vigilância sanitária são de competência exclusiva do Estado que, em benefício do interesse público atua com intervenções disciplinadoras das relações sociais produção-consumo em atividades de particulares e públicas que implicam risco à saúde coletiva ${ }^{1}$.

As ações e os serviços de saúde em suas variadas formas estão sujeitos à regulação sanitária, exercida fundamentalmente pela Vigilância Sanitária (Visa). Como braço do Estado, a Visa possui o atributo do poder de polícia ${ }^{2}$ e se organiza no Sistema Nacional de Vigilância Sanitária (SNVS) para desenvolver um conjunto articulado de ações nas três esferas de gestão. Estudos mostram irregularidades diversas em serviços de saúde ${ }^{3}$ e/ou seus produtos $^{4}$, serviços hospitalares ${ }^{5}$ e diagnósticos ${ }^{6,7}$ assim como em unidades básicas de saúde ${ }^{8}$, entre outros, que denotam muitas deficiências por parte dos prestadores de serviços e igualmente do sistema de controle sanitário.

Para o desenvolvimento das ações voltadas à proteção da saúde, a Visa ancora-se, principalmente, nos constructos teóricos de risco, segurança e qualidade em saúde, conceitos de difícil operacionalização nos serviços de interesse da saúde - onde ocorrem muitas incertezas. Os serviços de saúde são permeados por grande variedade de riscos, desde um conjunto de elementos relacionados à infraestrutura, adequação do ambiente, de instalações e equipamentos, práticas, procedimentos e tecnologias utilizadas.

Nos serviços de saúde, os riscos são de diversas naturezas, somam-se e são crescentes; principalmente em decorrência do desenvolvimento científico e tecnológico que produz continuamente novos produtos e em função da densidade tecnológica do serviço. São riscos relacionados a medicamentos e outros recursos terapêuticos, sangue, seus componentes e derivados, equipamentos, dispositivos médicos, saneantes etc., e relacionados também a diversos processos e práticas assistenciais realizados por distintos profissionais que, por diversas razões, cometem erros que resultam em eventos adversos que podem comprometer a segurança dos usuários.

Os efeitos negativos das tecnologias têm impulsionado preocupações, provocado mudanças em modelos organizacionais de controle sanitário em países desenvolvidos e colocado em alerta a questão da segurança sanitária ${ }^{9}$. Conceito em formação e valorização, segurança sanitária pode ser entendida como uma estimativa aceitável de uma relação risco/benefício atinente a um dado objeto de interesse da saúde. Os produtos e processos que estão sob vigilância sanitária portam riscos - razão desse controle - e pressupõem benefícios, exceto, é claro, os produtos do tabaco que, no Brasil são submetidos à vigilância sanitária. São riscos intrínsecos aos objetos e riscos adicionados ao longo da cadeia produtiva do objeto - da produção ao consumo ou na prestação do serviço; decorrentes de más práticas, de ilicitudes intencionais ou eventos fortuitos. Resta, ainda, a problemática do gerenciamento dos resíduos dos serviços de saúde com seus riscos para os trabalhadores da saúde, o meio ambiente e a população em geral.

A noção de qualidade pode ser compreendida como um atributo intrínseco, presumivelmente esperado de produtos e serviços relacionados com a saúde. A qualidade é de responsabilidade de produtores e prestadores de serviços, mas compete ao sistema público de saúde dispor de infraestrutura laboratorial, sistemas de notificação de eventos adversos e queixas técnicas, entre outros, e à vigilância sanitária cabe desenvolver estratégias e instrumentos para a verificação da qualidade dos bens fornecidos à população ${ }^{1}$ e realizar a vigilância dos agravos à saúde por eles produzidos, pois a qualidade de produtos e serviços integra os direitos do cidadão e do consumidor.

A atuação da Visa nos serviços de saúde, que antes ficava apenas a cargo dos estados, só passou a receber atenção da esfera federal após a criação da Agência Nacional de Vigilância Sanitária (Anvisa). Aquela atuação, junto com outros condicionantes, contribuiu para a consolidação de um modelo de controle sanitário de serviços de saúde bastante limitado, voltado à concessão da licença do estabelecimento, baseado apenas na inspeção sanitária $^{1,10}$. No geral, permanecem lacunas de ações que contribuam para a qualidade e segurança em saúde, evidenciadas pela escassez de indicadores que expressem a efetividade das ações ${ }^{10}$.

A despeito de constatados avanços ${ }^{11}$, a Visa, em especial nas esferas subnacionais, permanece com muitas fragilidades, notadamente no controle sanitário de serviços de saúde, uma tarefa desafiadora, sobretudo no ambiente hospitalar, onde múltiplos condicionantes contribuem para a ocorrência constante de erros e, assim, de eventos adversos. Um estudo com dados de 133 hospitais, tendo considerado durante um ano erros de diagnóstico, de dosagem ou de administração de 
medicamentos, uso incorreto de equipamentos, infecções hospitalares etc., revelou que 829 brasileiros morrem por dia em hospitais públicos ou privados devido a eventos adversos que comprometem a qualidade da assistência, causam sofrimento físico e psíquico de pacientes e aumento do custo assistencial. Em 2016, foram 302.610 óbitos $^{12}$.

Em face dos poucos estudos que tratam a temática de serviços de saúde e da vigilância sanitária e com o propósito de contribuir com o tema, este estudo se orientou pela seguinte pergunta: quais os principais desafios para a atuação da Visa nos serviços de saúde? Assim, tem-se por objetivo analisar os principais desafios à atuação dos trabalhadores de Visa nos serviços de saúde.

\section{MÉTODO}

Este estudo integra a pesquisa intitulada Vigilância Sanitária nos serviços de saúde da Atenção Básica ( $A B$ ) e Atenção Especializada (AE) em estados brasileiros, aprovada e financiada pelo Conselho Nacional de Desenvolvimento Científico e Tecnológico (CNPq), Edital Universal MCTIC/CNPq $n^{\circ} 28 / 2018$, cujo objetivo foi analisar o controle sanitário realizado pelas Visa estaduais e municipais em serviços de saúde na $A B$ e $A E$, tendo em vista a segurança sanitária e a proteção da saúde da população ${ }^{13}$. Tal pesquisa alcançou estados e municípios das cinco regiões brasileiras, com participação de trabalhadores da Visa e gestores das vigilâncias estaduais, coordenação da Atenção Primária estadual e de Hemocentros coordenadores.

Trata-se de um estudo de abordagem qualitativa ${ }^{14}$ que utilizou a técnica de Grupo Focal (GF) ${ }^{15,16}$ como estratégia para reunir trabalhadores de Visa e refletir sobre as ações de vigilância sanitária em serviços de $\mathrm{AB}$ e $\mathrm{AE}$, com liberdade e autonomia para realizarem seus relatos e depoimentos

O recrutamento dos participantes para os GF e a realização das sessões ocorreram durante o VIII Simpósio Brasileiro de Vigilância Sanitária/Associação Brasileira de Saúde Coletiva (Simbravisa/Abrasco), um evento de abrangência nacional, ocorrido na cidade de Belo Horizonte, no período de 25 a 27 de novembro de 2019. Os simposiastas ${ }^{\mathrm{a}}$ foram convidados de forma aleatória a participar dos GF, por meio de convites impressos, distribuídos nos auditórios e salas do Simbravisa, informando o objetivo da pesquisa, horário e local da realização dos GF.

Os dados foram produzidos em três sessões de GF, que contou com a participação de 22 profissionais, com duração média aproximada de $1 \mathrm{~h}$ para cada encontro, realizadas em salas disponibilizadas pela organização do Simbravisa, com cadeiras dispostas em formato de círculo. A todos que participaram foi garantida a fala no debate, inclusive por meio de perguntas que se direcionavam àqueles(as) que porventura não tivessem se manifestado sobre algum ponto de discussão do GF. No entanto, foram observadas diferenças nos tempos de fala pelos participantes de alguns estados, o que pode ser explicado pela composição dos GF (ver Quadro 1), ainda que o conteúdo dos três encontros tenha circundado os mesmos temas.

No primeiro encontro (GF1), os participantes dos estados do Nordeste (NE) e Sul (S) apresentaram maior tempo de fala, o que pode ser justificado pela diferença entre o número de participantes por estado - NE1 (quatro), NE3 (dois) e S1 (dois), e apenas um participante de NE2 e outro de S2. No segundo encontro (GF2), os maiores tempos de fala foram utilizados por SE1 e NE3, com três e dois participantes respectivamente. No terceiro e último encontro (GF3), embora quatro profissionais de $\mathrm{S} 1$ tenham participado, houve equilíbrio na participação e tempo de fala com os participantes de N1 e NE4.

As sessões foram conduzidas por uma moderadora e três ou quatro observadoras que participaram de todos os GF, sem qualquer relação de hierarquia ou subordinação entre equipe de pesquisa e participantes. Foram realizados os registros em diário de campo sendo eles utilizados para subsidiar as análises e a interpretação do material. Todas as ações foram orientadas por um roteiro com quatro questões sobre: as atividades de Visa desenvolvidas nos serviços de saúde; as ações de Visa desenvolvidas em serviços hemoterápicos; as ações desenvolvidas pela Visa na $A B$ e as condições de constituição e funcionamento dos Núcleos de Segurança do Paciente nos serviços da AB e AE (Quadro 1).

Quadro 1. Composição dos grupos focais, segundo número de participantes e regiões do Brasil. Belo Horizonte, 2019.

\begin{tabular}{|lcc|}
\hline Data do grupo focal & Número de participantes & Número de estados por região do Brasil \\
\hline GF1 $(25 / 11 / 2019)$ & $10:$ & 3 estados do Nordeste (NE) \\
NF2 $(26 / 11 / 2019)$ & 2 estados do Sul (S) & 2 estados do Nordeste* \\
& NE1 (1), NE3 (2), SE1 (3) NE3 (2), S1 (2), S2 (1) & 1 estado do Sudeste (SE) \\
GF3 $(27 / 11 / 2019)$ & Ne: & 1 estado do Norte (N) \\
& N1 (1), NE4 (1), S1 (4) estado do Nordeste & 1 estado do Sul*
\end{tabular}

Fonte: Elaborado pelas autoras, 2021.

GF: grupo focal; NE: Nordeste; S: Sul; SE: Sudeste; N: Norte.

Os diferentes números que compõem cada sigla significam os diferentes estados da mesma região, por exemplo: NE1, NE2, NE3, NE4: quatro diferentes estados da região Nordeste. E o número entre parênteses, após a sigla, por exemplo: NE1(4), corresponde ao número de participantes, neste caso quatro, por estado/região/grupo focal.

* Estados representados em mais que um encontro do GF. 
A composição dos GF contou com profissionais de formações diversificadas na área da saúde e afins (enfermagem, odontologia, medicina, medicina veterinária, direito e física), de oito estados e quatro regiões brasileiras. Todos os participantes assinaram um Termo de Consentimento Livre e Esclarecido e tiveram as falas registradas em equipamento de áudio digital, posteriormente transcritas integralmente. As transcrições foram realizadas por participantes da pesquisa na condição de bolsistas voluntários, graduandas de farmácia e saúde coletiva ou residentes de planejamento e gestão. Não houve registro do tempo de transcrição dos GF, entretanto todas as transcrições foram revisadas pelos pesquisadores do grupo.

Os registros e as transcrições foram tratados segundo a técnica de análise de conteúdo temática ${ }^{14}$. A pré-análise constou da leitura flutuante e categorização dos dados por quatro pesquisadoras, individualmente; em seguida, procedeu-se à categorização, coletivamente. Esta etapa possibilitou a agregação em unidades de análise, com a descrição das características do conteúdo dos dados, que foram agrupados em duas categorias temáticas: Gestão e Organização dos Serviços e Condições de Trabalho, e, posteriormente, a identificação de subcategorias, conforme apresentado no Quadro 2, de modo que permitissem aproximações com o objetivo do estudo. Os achados relacionados a tais categorias e subcategorias circundaram a discussão nos três encontros de GF.

Esse projeto foi aprovado pelo Comitê de Ética do Instituto de Saúde Coletiva da Universidade Federal da Bahia, conforme pareceres $\mathrm{n}^{\circ} 3.423 .630, \mathrm{n}^{\circ}$ 3.819.357 e CAAE 12491019.2.0000.5030.

\section{RESULTADOS E DISCUSSÃO}

As categorias temáticas e subcategorias são resultantes da classificação do corpus produzido pelos trabalhadores de Visa participantes dos GF (Quadro 2). Os participantes não circunscreveram suas falas às questões postuladas no roteiro e abordaram os mais diversos problemas e desafios que enfrentam na realização de suas atividades. No intuito de manter o anonimato dos participantes dos GF, os excertos utilizados foram classificados de forma aleatória por meio de registro alfanumérico.

Gestão e Organização dos Serviços

A categoria Gestão e Organização dos Serviços de Visa agrupou a maior parte das falas que expressaram desafios. Relacionado a esta categoria, identificou-se um conjunto de temas que sobressaíram nos discursos dos participantes dos GF e foram agrupados nas subcategorias temáticas: planejamento, organização do trabalho, coordenação e integração das ações, rotatividade de gestores e uniformização de procedimentos.

No referente ao planejamento das ações, a fala dos participantes descreve uma prática incipiente e, quando existe, é desconsiderada, em decorrência das demandas urgentes priorizadas pela gestão. Os participantes ainda atribuem à excessiva demanda, feita pelos gestores internos e pelo Ministério Público, as dificuldades em seguir a programação de trabalho, como mostram os excertos a seguir:

[...] o planejamento em nenhum momento vai acontecer quando a coisa é pra apagar incêndio, porque é uma espécie de incêndio isso, né? porque você não está lá planejando essas inspeções, ela simplesmente aconteceu e você se depara nessa realidade, com essa diferença (NE1, GF1).

[...] uma responsabilidade que a gente chama atenção, "não existe a questão planejamento", isso é bem claro, existe, sim, a denúncia, convocação do Ministério porque quer relatório ou ouvidoria [...] (NE3, GF1).

A gente trabalha com planejamento, que é uma escala mensal, onde a gente recebe a demanda, só que as demandas são muito grandes, como eu já disse desde o começo. A gente trabalha muito mais pra o Ministério Público que pra a licença, então a gente tem uma escala mensal onde a gente coloca as prioridades, mas existem momentos que a gente não consegue fazer, cumprir esse planejamento porque existe as demandas de urgências e emergências, então a gente precisa mudar nossa programação (NE3, GF2).

Isso interfere bastante, final do ano que tem uma demanda toda reprimida, sempre que tem que encerrar as metas a gente está praticamente atendendo Ministério Público e solicitação de gestores. E aí fica prejudicado o planejamento, eu sou do nível estadual, então a gente não tem essa articulação com o município, com o planejamento com o município, fica a nível estadual mesmo e os municípios fazem seu planejamento independente (NE4, GF3).

Quadro 2. Categorias e subcategorias temáticas relacionadas aos principais desafios à atuação dos trabalhadores de Vigilância Sanitária nos serviços de saúde.

\begin{tabular}{|lc|}
\hline Categorias temáticas & Subcategorias \\
\hline Gestão e Organização dos Serviços & Planejamento das ações \\
& Organização do trabalho \\
& Coordenação e integração das ações \\
& Rotatividade de gestores \\
Condições de Trabalho & Uniformização de procedimentos \\
& $\begin{array}{c}\text { Redução do quantitativo de trabalhadores } \\
\text { Precarização dos vínculos de trabalho, situação salarial }\end{array}$ \\
\hline
\end{tabular}

Fonte: Elaborado pelos autores, 2021. 
[...] a gente não trabalha com ações, a gente trabalha com demandas, do Ministério Público, demandas de rotina; eu como fiscal, eu recebo minha rotina, eu tenho uma comanda de escalas [...] então a gente consegue trabalhar de maneira organizada, é lógico que como qualquer serviço sempre falta $\mathrm{RH}$, porque a demanda do Ministério Público [...] (N1, GF3).

[...] a gente faz ações de controle, apura também as denúncias tanto de Ministério Público quanto aquelas que chegam via ouvidoria ou via Conselho, elas correspondem a uma parte muito grande do nosso universo de ação, o Ministério Público [...] (SE1, GF2).

O planejamento e a avaliação em vigilância sanitária ainda não foram incorporados, tampouco o uso de indicadores - necessários ao diagnóstico e avaliação da área - que podem permitir a análise da situação de saúde e o conhecimento dos fatores determinantes e condicionantes ${ }^{17,18,19}$, especialmente aqueles relacionados ao campo específico de atuação da Visa. No estudo de Maia e Guilhem ${ }^{20}$, a ausência de indicadores e ferramentas para avaliação das ações de vigilância sanitária constituiu um dos cinco principais desafios à regulação sanitária brasileira como parte da política de saúde. No presente trabalho, a análise das falas sobre o planejamento denota que se trata de programação de atividades e não exatamente de planejamento de ações.

Em um estudo de casos de Visa estaduais ${ }^{21}$ foi constatado que os serviços estudados não realizam planejamento das ações de vigilância sanitária nos serviços de saúde, nem do reúso de produtos médicos de uso único; e não aplicam nenhum método de avaliação do reúso e nem realizam capacitação específica dos seus profissionais.

O Relatório do Fórum Nacional ${ }^{22}$ que encerrou os fóruns regionais do Ciclo de Debates em Vigilância Sanitária, que contaram com ampla participação de trabalhadores e gestores da área, acadêmicos e outros convidados relacionou os 12 principais desafios discutidos nos eventos e considerados prioritários, e ainda as propostas para seu enfrentamento. Entre esses desafios, encontram-se a falta de planejamento e o estabelecimento de instrumentos pré-definidos no processo de descentralização, considerado precário e fragilizado, posto que não leva em conta a realidade e a diversidade local, o que dificulta a pactuação entre os componentes do SNVS.

Sobre o processo de descentralização, os participantes dos GF deste estudo discutiram as dificuldades identificadas nas pactuações e a rotatividade dos gestores, o que, no entendimento dos participantes, acaba prejudicando a consolidação do SNVS no seu braço estadual, como ilustram os excertos abaixo:

[...] acho que a gente teve piora, porque quem tinha já, a 207 [RDC n²07, de 3 de janeiro de 2018 - Anvisa] diz que você tem que pactuar, então não era pra atuar, então você simplesmente tinha que descentralizar os serviços. Aí, como a 207 disse que baixo risco era pra eles assumirem, então se eles pegavam algum de alto [risco] eles já devolviam pra o estado. Tem que pactuar, tipo assim, não querem assumir. De fato, os municípios têm muita dificuldade, porque tem a rotatividade, eles acabam chegando novos, aí, e já que não é minha obrigação, não tá escrito lá que eu tenho que ser do alto [risco], então deixa pra o estado (NE4, GF3).

Vejo um esfacelamento do sistema de vigilância como um todo e no estado [...] houve um esvaziamento que compromete totalmente esse controle de riscos, e é extremamente preocupante (NE1, GF1).

A classificação dos estabelecimentos como sendo de baixo, médio e alto risco foi uma estratégia utilizada para demarcar competências no processo de descentralização e para operar uma racionalidade na organização do processo de trabalho nos serviços de Visa. Contudo, segundo os debates ocorridos nos Fóruns, as Visa estaduais têm assumido as inspeções de médio e alto risco, dado que ainda se observam grandes dificuldades de infraestrutura dos serviços municipais para atuarem em áreas consideradas de médio risco ${ }^{22}$. Os excertos a seguir abordam tais aspectos:

[...] a gente não consegue atingir toda a demanda. Assim, $100 \%$ do universo que a gente deveria estar inspecionando, a gente não consegue. 0 quadro de servidores hoje não é compatível para atender a toda essa demanda. Então, a gente trabalha com uma priorização e uma categorização de risco $[\ldots]$ (SE1, GF2).

[...] um serviço mais básico, digamos, consultório médico, que a gente vai observar, consultório odontológico que na classificação de risco seria alta, mas o município, com uma equipe mínima ele teria condição de fiscalizar. E nós não temos muito esse apoio, só municípios com mais de 100 mil habitantes que eles têm estrutura pra inspecionar a Atenção Básica, ou seja, pouquíssimos no estado (NE4, GF3).

Recentemente a gente fez uma oficina envolvendo as vigilâncias sanitárias, as vigilâncias municipais e as regionais, pra que se a gente começar aquele processo de descentralizar, primeiro a gente vai capacitar, fazer um trabalho de parceria, pra depois a gente iniciar os hospitais já que eles não ficam com hospitais de maior complexidade, pelo menos os de baixa e média, pra que eles consigam pelo menos ser nossos parceiros, porque sozinha a gente não tem condições, é muita coisa (NE3, GF2).

Conceito central na atuação em vigilância sanitária, o risco permanece como um constructo pouco trabalhado, sujeito às veleidades de um processo de lenta construção teórico-conceitual e doutrinária na área. Conforme o Relatório do Fórum Nacional ${ }^{22}$, outro desafio considerado prioritário para o SVNS e para a população é a inexistência de harmonização e entendimento sobre risco.

Entretanto, nas falas dos participantes dos GF não emergiu nenhuma problematização neste sentido; o uso da categoria risco apareceu sempre associado às definições existentes nos regulamentos e, especificamente no tocante à classificação dos estabelecimentos sob vigilância sanitária, como de baixo, médio e alto risco. Observou-se também, que, em face das dificuldades 
decorrentes da escassez de trabalhadores, de recursos materiais e tecnológicos, os profissionais declaram atuar centrados no que é definido como atividades de alto risco.

[...] trabalha em cima de risco, ele indica se é baixo a alto risco, então é meta prioritária também da Anvisa e a gente seguiu esse caminho, os estabelecimentos de alto, médio e alto risco serem prioridades [...]. Então, mas a gente não está conseguindo dar conta disso pela deficiência de recursos humanos, isso está sendo gravíssimo no nosso estado, e não sei como é o que o estado vai solucionar esse problema a curto, médio e longo prazo, mas é a situação crítica que a gente não está dando conta mais desse planejamento, de assumir mais essas metas (NE1, GF1).

[...] então, lá o estado fica com média e alta complexidade, que a gente chama, que envolve os serviços de internação, com maior complexidade mesmo, né? (NE3, GF2).

[...] porque o estado já tem alta complexidade, não consegue descentralizar, é um desafio enorme porque, não sei no estado de vocês, mas no XXX, quando fala em serviços de saúde [...] a vigilância treme do município, acha impossível assumir os serviços de saúde [...]. Então temos essa dificuldade (NE4, GF3).

Além de repetidos problemas de gestão e, muitas vezes, da fragilidade organizacional e da insuficiência da estrutura institucional para resolvê-los, os participantes aventaram conflitos de interesse na condução da fiscalização em alguns municípios:

[...] quando você coloca o fiscal da prefeitura pra inspecionar a Unidade de Saúde de certa forma as pessoas vão cobrar dele como trabalha algo, e se depara com uma situação conflituosa mesmo, né? $\mathrm{E} X$ também tem essa característica, porque o fiscal ele é concursado do cargo específico de fiscalização e ele está impedido supostamente de ir para a assistência, então ele não tem nível de fiscalizar ele mesmo em outro horário, às vezes tem, mas não é pra acontecer, né? Agora os colegas dele da assistência, como é que você conduz o processo de fiscalização se você tem interesse fundamental [...] (NE1, GF1).

Reitera-se que as questões relacionadas ao trabalho no SUS ainda não receberam a prioridade de tratamento que lhes é devida e, na Visa, a situação é agravada pela natureza do trabalho que impõe limitações de atuação aos seus servidores, por razões de conflitos de interesse. Como agentes do Estado na função regulatória, não podem ter vínculos empregatícios concomitantemente com segmentos regulados. As ações de vigilância sanitária são de competência exclusiva do Estado e, enquanto Administração Pública, a Visa está submetida a limitações no exercício de direitos individuais em benefício do interesse público, de modo que seus trabalhadores não podem exercer, ao mesmo tempo, função em entidade pública e privada nas mesmas áreas de atuação ${ }^{23}$.

Outro tema levantado pelos participantes dos GF, relacionado à gestão e organização da Visa, foi referido como dificuldade na coordenação e integração das ações entre as diferentes instâncias de gestão. Isso foi especialmente observado quando os participantes tratavam das questões de segurança do paciente na Atenção Primária à Saúde e sobre ações que sofreram alteração de competência da esfera estadual, no processo de descentralização. Os excertos seguintes ilustram a questão:

[...] a política de Segurança do Paciente, ela tem uma portaria do Ministério da Saúde e ela tem uma RDC [Resolução de Diretoria Colegiada] da Anvisa. Então, parece que são duas coisas que andam separadas, mas elas tem que andar juntas. Mas a representação federal, estadual e do município tem que ser, eu não vejo dois galos no mesmo galinheiro. Então tem que ser um só, pra conduzir essas estratégias, eles vão fazer um evento e tipo assim, a gente tem que correr atrás deles pra participar, senão a gente fica assim desagregado (NE4, GF3).

A gente sofreu muito isso [sobre coordenação de ações em diferentes instâncias da gestão] quando houve o começo da descentralização, porque a gente percebia que o estado estava muito apegado em alguns serviços e não queria largar, né? Então a gente percebeu, mas agora, depois de alguns anos, a gente não percebe mais. [...] a gente no começo, tinha essa questão de dois galos no mesmo galinheiro, porque a gente, eles da Visa estadual achavam que eram os donos do processo, mas com o tempo a gente foi mostrando pra eles que éramos parceiros, e hoje a gente trabalha em parceria (N1, GF3).

Distanciamento, falta de articulação e de interlocução sistemática e desarmonia entre os entes do SNVS compõem outro desafio, considerado prioritário no Relatório do Fórum Naciona ${ }^{22}$ que interfere na atuação da Visa, nos seus resultados. Sinalize-se, ainda, a falta de integração das ações de vigilância sanitária nas políticas de saúde em geral. Conforme o estudo de Maia e Guilhem ${ }^{20}$, o desconhecimento da função da vigilância sanitária na saúde é mais um dos principais desafios à regulação sanitária no Brasil.

Conquanto formalizado pela Lei $\mathrm{n}^{\circ} 9.782$, de 26 de janeiro de 1999, o SNVS persiste com um vazio quanto à configuração e organização, caracterizado como um sistema fragmentado apenas o componente federal foi reformado estruturalmente - revelando-se fragilmente estruturado em termos de laços de cooperação e responsabilidades, além de eficácia relativa ${ }^{11,24}$.

É preciso atentar que um conjunto de atribuições são compartilhadas entre as três esferas de gestão, o que denota uma clara interdependência entre os componentes do SNVS e intercomplementariedade das ações. Para o cumprimento de certas competências, como: registro de produtos, autorização de funcionamento de empresas, por exemplo, a Anvisa requer a licença sanitária dos estabelecimentos, cuja concessão é de responsabilidade de estados ou municípios. No caso de serviços de saúde, a licença sanitária é o primeiro requisito para hospitais participarem de processos de acreditação, entre outros. A interdependência em atribuições legalmente estabelecidas, como sinalizam Patrício et al. ${ }^{25}$, interfere na qualidade das ações realizadas pelos órgãos de Visa. 
A efetiva qualificação das ações de vigilância sanitária fortalece os processos de construção coletiva, uma vez que está associada à definição das responsabilidades, fundamentada em critérios técnicos de competência e na uniformidade na execução de suas ações, com impacto na proteção da saúde da população ${ }^{26}$.

Outro tema abordado pelas participantes dos GF foi a rotatividade de gestores. A insatisfação com as mudanças frequentes de gestores emerge nas falas como um fato que dificulta a atuação da Visa, haja vista que implicam descontinuidade do trabalho: "[...] a gente também sofreu um processo de numa gestão, quatro diretores mudaram, a cada direção que muda, volta à estaca zero do que está sendo construído" (NE1, GF1).

Para os entrevistados, a rotatividade de gestores também resulta em sobrecarga de trabalho para a Visa estadual, uma vez que não há entendimento, por parte dos gestores recém-chegados à esfera municipal, sobre a pactuação, no referente aos estabelecimentos classificados como de alto risco, conforme a Resolução da Diretoria Colegiada (RDC) da Anvisa $n^{\circ} 207 / 2018$, como se nota no excerto a seguir:

[...] os municípios têm muita dificuldade, porque tem a rotatividade [falando dos gestores], eles acabam chegando novos, aí, [...] já que não é minha obrigação, não tá escrito lá que eu tenho que ser do alto [risco], então deixa pra o estado (NE4, GF3).

Além da descontinuidade, os participantes atribuem à rotatividade a existência de um sentimento, entre os gestores, de não pertencimento ao grupo de trabalho, o que pode resultar em desresponsabilização pelos processos em curso.

[...] porque a gente entende que se eles não se sentirem parte do processo as coisas não vão acontecer, e, às vezes, quem está numa regional com a realidade diferente, ele vai poder dizer. Isso, dessa forma, não funciona para a gente (NE1, GF1).

Problemas relacionados à ausência de gestão profissionalizada, como ressaltam autores ${ }^{27,28}$, podem comprometer a qualidade dos serviços no SUS, tanto pela inexistência de profissionais com qualificação para a realização de atividades específicas e complexas, quanto pela interferência causada pelas indicações políticas para os cargos e funções de direção em todos os níveis do sistema público de saúde. Principalmente no âmbito dos municípios, a Visa se depara com um conjunto de dificuldades relacionadas a interferências político-partidárias e à falta de apoio político que se somam à desmobilização e desinformação da sociedade ${ }^{29}$.

A questão da UBS a gente lá em XXX [capital do estado] só faz via demanda do Ministério [...], são ligados à prefeitura Municipal e isso gera um problema [...], aí a gente fica ad eterno indo fiscalizar o serviço e muitas das vezes, faz relatório e continua a inadequação. Inclusive eu estava até conversando com a minha gestora porque eu pedi pra interditar uma UBS, sem condições de funcionamento a UBS por falta de higiene mesmo, por falta de gerenciamento mesmo [...], então eu solicitei interdição $[\ldots]$, quando eu retornei lá dois meses depois o que eles fizeram foi demitir o diretor, quer dizer o que estava pior ficou péssimo [...], eu fui lá, piorou e sem diretor, aí ela me falou que como é uma instituição que está ligada à Secretaria de Saúde fica difícil muitas vezes (N1, GF3).

[...] a gente faz os relatórios, entrega pra o gestor e fica do gestor estipular o que ele vai fazer, então, na maioria das vezes, é engavetado e não há melhorias (S1, GF3).

$\mathrm{O}$ alinhamento de procedimentos foi outro assunto abordado nos GF. Na opinião dos participantes, alinhamento e uniformização de procedimentos constituem uma estratégia importante para a gestão e organização dos serviços, dado que contribuem para assegurar aos gestores um sentido de unicidade e pertencimento ao processo. Ou seja, contribuem para que eles se sintam parte do grupo e, dessa forma, os problemas podem ser melhor autorreconduzidos.

Segundo os participantes, as regionais e os distritos sanitários precisam estar trabalhando em sinergia com suas bases, mas, também, de maneira padronizada como um todo, sem desvalorizar nem deixar de atender às necessidades regionais/locais:

[...] a gente fez revisão desses POP [procedimentos operacionais padronizados] com base nisso para atingir o todo. Isso está sendo um trabalho que realmente vem dando resultado; então, é uma forma também da gente conseguir padronizando as ações; a gente está construindo um mesmo objetivo (NE1, GF1).

[...] então nós temos regionais que tem 60 municípios, temos regionais que abrangem 20 municípios. Então, essas regionais que têm dentro da sua estrutura um núcleo de vigilância e é composto por várias categorias profissionais e então eles demandam essas atividades, esses serviços que seriam de responsabilidade do estado (S2, GF1).

A questão da padronização dos procedimentos em vigilância sanitária, assim como a qualidade das ações, não é simples, de fácil equacionamento. Sem minimizar as dimensões de incertezas e subjetividades inerentes às áreas de regulação e gerenciamento de riscos, remetem tanto à formação, qualificação, quantitativo dos profissionais e modalidades de inserção, como também se relacionam com a infraestrutura e organização institucional, a disponibilidade de recursos tecnológicos ou meios de trabalho adequados e suficientes. Em suma, vinculam-se a uma política pública que priorize a proteção da saúde no escopo da vigilância sanitária.

Ao problematizarem um questionamento apresentado pela Central dos Hospitais de Minas Gerais sobre a falta de padronização do trabalho da Visa, Oliveira e lanni ${ }^{30}$ defenderam que

a padronização e a qualificação das ações de fiscalização da Visa estão diretamente relacionadas à existência de uma Política Nacional de Educação Permanente em Visa, que promoverá o acesso a conhecimentos científicos pertinentes às ações de regulação, bem como ao entendimento crítico 
do real sentido do Poder de Polícia pelos trabalhadores do SNVS. Relacionam-se, também, com uma infraestrutura satisfatória e recursos humanos em número adequado para a atuação da Visa, afinal, é a sua força de trabalho quem the assegura qualidade e efetividade ${ }^{30}$.

\section{Condições de Trabalho}

A categoria Condições de Trabalho agrupou um conjunto de temas que se entrelaçam como desafios à atuação dos trabalhadores de Visa: redução de pessoal, precarização dos vínculos de trabalho, situação salarial, disponibilidade de recursos tecnológicos para a realização das ações. Os temas estão inter-relacionados, mas optou-se por organizar os relatos separadamente, visando identificar os desdobramentos, o movimento dos trabalhadores e as resultantes que essas condições de trabalho têm produzido sobre a Visa.

Os profissionais expressaram grande preocupação com a redução dos quadros de trabalhadores que vem ocorrendo ao longo do tempo nos serviços de Visa. Em geral, as falas atribuíam tal fato a aposentadorias, relocação de servidores para outras áreas devido a mudanças na estrutura administrativa nos estados, o que vem tornando as remunerações pouco atrativas. Soma-se, ainda, a não realização de concursos públicos para incorporação de novos servidores de modo a recompor os quadros. Ademais, o contexto de grave crise econômica dos estados tem contribuído para atrasos e parcelamentos de salários dos servidores e ainda para a redução de direitos.

[...] eu não vou nem falar na nossa situação porque é de chorar. Primeiro a situação salarial, 47 meses com o salário parcelado; o salário de outubro a gente nem recebeu ainda, a gente vai quitar o salário de outubro em dezembro, aí vai pagar a segunda ou terceira parcela, não sei nem... enfim, melhor nem falar, que é triste [...] (S2, GF1).

[...] o novo governo assumindo extinguiu 31 diretorias regionais e transformou em nove núcleos regionais de saúde. Isso fragilizou bastante [...] a nossa capilaridade, a nossa atuação nas regionais e a perda de insalubridade também, essas reformas administrativas, [...] esse projeto de aposentadoria [...]. Então, durante esses anos, eu tenho percebido um esvaziamento tão grande que compromete todo o planejamento (NE1, GF1).

Então, 50\% mais ou menos de aposentadoria da equipe estadual, né, nossa, inclusive nas regionais, então aquele grupo que foi falado aqui também, que tinham um grupo, os antigos e tal, a gente não tem mais [...] de tudo e que a gente tá tentando dar conta, mas a gente não dá, e o mínimo, e a gente quase não está fazendo, o nível central não está fazendo inspeção do sangue porque a gente não tem condições mais com essa equipe tão reduzida (S1, GF1).

No estado XXX existe uma divisão dos serviços de saúde, onde está acoplada a essa divisão dos serviços de saúde [...]. É uma equipe multidisciplinar, porém pequena. Concursado mesmo a gente tem pouco. A gente tem também os contratos, então é bem difícil, a demanda é muito grande [...] (NE3, GF2).

O cenário atual de incertezas e de redução e perda de direitos dos servidores públicos provocou um aceleramento de pedidos de aposentadorias que contribuem para a redução do quantitativo de trabalhadores. A situação tem-se agravado, segundo os participantes, por ausência de recomposição dos quadros de servidores e consequente prejuízo na qualidade das inspeções, segundo os quais os serviços de Visa contavam com servidores qualificados, com expertise em certas áreas, como por exemplo, no caso dos profissionais que inspecionavam os serviços hemoterápicos:

[...] na regional que eu trabalhava, a gente ficava responsável, hoje essa regional é responsável por 72 municípios, então, a equipe que era multidisciplinar fazia toda a média e a alta complexidade. Só que a gente, nesse mesmo período, passou por uma transição, assim, muito desafiadora, porque o grupo que a gente tinha intitulava GT SANGUE, foi totalmente dissolvido; era um grupo [...] composto em 2012, pra vocês terem noção, [...] por 26 membros pra fazer inspeção em hemoterapia [...] (NE1, GF1).

E a gente também teve um fator muito marcante pra gente dentro da coordenação de serviços de saúde, porque nós tivemos um êxodo de servidores, muitos servidores acabaram evadindo da Vigilância Sanitária. Eu acredito que não é diferente dos outros estados, mas a gente recebeu muita gente da assistência, como a gente não tem previsão de concurso até 2023, por conta do regime de recuperação fiscal, então a gente não tem a perspectiva de renovação do quadro, então essas pessoas foram muito bem recebidas, porque de fato elas são importantes pra a gente, né. Só que elas vieram sem a cultura da Vigilância Sanitária e também sem a cultura da Segurança do Paciente, então esse é o desafio [...] (SE1, GF2).

A infraestrutura inadequada para a atuação da Visa, a escassez de profissionais, a sobrecarga de trabalho foram temas discutidos no Ciclo de Debates e reconhecidos como desafios a serem priorizados ${ }^{22}$.

A investidura pública de profissionais de distintas formações, com qualificação e consciência da responsabilidade no exercício de suas funções, aliada a uma política de recursos humanos que inclua o aumento, a qualificação e a estabilidade profissional constituem condições fundamentais para o fortalecimento da Visa em todos os municípios do Brasil ${ }^{29}$ como também em todos os estados. Silva et al. ${ }^{11}$ acrescentaram a importância do debate sobre carreiras para a força de trabalho da Visa nos estados e municípios, além da necessidade de ampliar o quantitativo e reforçar a qualificação dos trabalhadores com enfoque na atuação sobre os riscos à saúde nos diferentes territórios ${ }^{11}$.

A implementação de uma política capaz de atender as demandas do trabalho em saúde é desafiadora. Soma-se a falta de atenção e de capacidade dos governos para investir em infraestrutura que possa melhorar as condições de trabalho no SUS. Melo et al. ${ }^{31}$ chamaram a atenção que a posição das organizações influencia o comportamento dos trabalhadores; quando esta 
posição é afirmativa, pode envolver, por parte dos trabalhadores, sentimentos de pertencimento e satisfação com o trabalho que realizam e o desejo de permanecer e continuar no emprego. Os autores concluem que os gestores de Visa devem considerar a importância dos seus trabalhadores na implementação da política de saúde e priorizar ações que resultem em níveis elevados de comprometimento desses profissionais.

Nos GF deste estudo, outro tema que se destacou nas falas dos participantes, por refletir nas condições de trabalho, está relacionado à (in)disponibilidade de recursos tecnológicos para a realização das atividades e ainda de sistemas de informação que se comuniquem, questão também elencada entre os desafios prioritários discutidos no Ciclo de Debates ${ }^{22}$.

O tema sistemas de informação foi realçado como ferramenta importante para gerenciar as ações e assegurar melhores condições de trabalho. Chama a atenção que apenas estados das regiões Sul e Sudeste referiram trabalhar com versões atualizadas de sistemas de informação que realmente davam suporte e informações para o planejamento e a execução das atividades.

A gente já tem uma série de sistemas, né? Apoiando essas ações todas, hoje o processo de licenciamento já é todo digitalizado, todo informatizado, o sistema denominado protocolo online, [...] a partir daí a gente tem as ações do controle e regulação. $E$ também tem alguns sistemas que funcionam como um apoio interno nosso, mas são sistemas, mas de gerenciamentos de autos de infração, pra acompanhar os processos administrativos sanitários, e diria que basicamente é essa nossa estrutura (SE1, GF2).

[...] a gente tem um sistema também [...] que não são todos os municípios que aderiram [...] porque alguns municípios já tinham há muitos anos [...] mas é um sistema onde o município entra nesse sistema, pede apoio e nós, a regional entra no sistema e vê quais foram as inspeções, que está sendo pedido apoio, e a gente também no central vê $[\ldots](\mathrm{S} 1, \mathrm{GF} 1)$.

Nós temos também um sistema que vai ajudar a gente na parte de planejamento [...] que é um sistema de vigilância que é pra emissão de alvará, dados de informação, cadastro, controle de serviços e que a gente tem uma portaria estadual que obrigatoriamente os municípios têm que aderir aquele sistema, então a gente está com $70 \%$ dos municípios já aderiram a esse sistema do estado e a gente consegue fazer todo o monitoramento [...] (S2, GF1).

Em geral, entre os participantes do Nordeste, a informação sobre o uso de tais sistemas de informação é que ainda se encontravam em fase inicial ou são subutilizados, o que demonstra desigualdade na infraestrutura dos serviços em detrimento de condições de trabalho adequadas às funções de Visa.

[...] eu acho que essa parte documental a gente ainda é um pouco bem incipiente. Não temos um sistema informatizado, nem só contamos mesmo imprimir lá os checklists e guias de inspeção [...] A gente tem um sistema de inspeção [...] que a gente alimenta todas as informações, mas é agora, esse ano ainda vai haver um treinamento, porque algumas pessoas saíram, outras chegaram e tudo, a gente precisa ter essa capacitação. [...] é um retreinamento de novo, né? e vai ser colocado em prática. É um sistema que a gente vai alimentar, as inspeções, como é que a gente fez, se o hospital está apto a receber a licença ou não, entendeu? É um sistema bem interessante lá (NE3, GF2).

A ausência de um sistema de informação em nível nacional constitui um problema estrutural do SNVS; contribui para a falta ou precariedade de informações relativas e atualizadas sobre os segmentos regulados, sobre as ações de fiscalização e sobre os processos administrativos; traz dificuldades no monitoramento dos serviços e na identificação de eventos adversos ${ }^{29}$, também nos processos de tomada de decisão, e comprometem a formulação de política consistente com a realidade local ${ }^{25}$. Portanto, um sistema de informação específico para a Visa, com indicadores de base técnico-científica, representativa do trabalho desenvolvido, possibilita obter informações úteis aos processos de transformação da realidade. Um desafio é formular um sistema de informação alinhado com a finalidade das ações ${ }^{17}$. A ausência de um sistema de informação, identificado como um dos principais desafios à regulação sanitária no $\mathrm{Brasil}^{20}$, permanece, mesmo após mais de 20 anos da criação da Anvisa que tem entre suas competências institucionais, coordenar o SNVS.

A reflexão sobre a vigilância sanitária, cujas ações são de natureza essencialmente preventiva, sublinha sua relevância para a saúde e a necessidade do seu fortalecimento técnico e político. Lucchese $^{32}$ ressaltou esse componente do SUS como um espaço privilegiado de intervenção do Estado, pois funções e instrumentos que são inerentes à Visa possibilitam uma atuação de modo a elevar a qualidade de produtos e serviços relacionados com a saúde e ainda adequar os respectivos segmentos produtivos e os ambientes às necessidades do sistema de saúde e às demandas sociais em saúde.

\section{CONCLUSÕES}

O estudo revelou uma série de desafios para o desenvolvimento das ações de controle sanitário nos espaços operativos do SNVS e evidenciou importantes temas que podem ser objetos de novas investigações, como: a persistência de problemas relacionados à organização e gestão dos serviços, a exemplo da incipiente prática de planejamento das ações; a falta de articulação e interlocução sistemática e a desarmonia entre os entes do SNVS; a fragilidade organizacional e insuficiente infraestrutura e de pessoal, entre outras questões. As dificuldades encontradas na organização dos serviços de Visa e nas condições de trabalho certamente se refletem no controle sanitário dos serviços de saúde e, por conseguinte, na qualidade destes serviços e na segurança sanitária dos usuários.

Algumas diferenças regionais foram observadas no que diz respeito ao uso de sistema de informação para atuação da Visa, com destaque positivo para estados das regiões Sudeste e Sul e dificuldades expressadas pelos trabalhadores de estados das regiões 
Norte e Nordeste. O conceito de risco, construto fundante das ações de vigilância sanitária, continua a ser um processo de lenta construção teórico-conceitual e, consequentemente, de difícil operacionalização no controle sanitário de serviços de saúde.

Observou-se que temas relacionados às precárias condições de trabalho foram fortemente destacados pelos trabalhadores de Visa participantes dos GF. A conjuntura política e econômica mais recente no Brasil levou ao subfinanciamento do SUS e à agudização de problemas estruturais crônicos da organização e funcionamento da Visa no Brasil. Percebeu-se que o trabalhador da Visa atua em um ambiente de insatisfação devido às condições salariais e de trabalho e à acentuada perda de direitos gerada pelas reformas previdenciárias em alguns estados e, ainda, pela ausência de concursos públicos para recomposição das equipes de trabalho.

Diante das dificuldades e dos desafios apontados pelos trabalhadores de Visa para o pleno desenvolvimento de suas atividades, cabe refletir como estas fragilidades apontadas podem repercutir nos indicadores de qualidade e segurança sanitária nos serviços de saúde ofertados à população. Cabe, portanto, reafirmar a importância estratégica destes serviços na proteção da saúde pública.

Os desafios são múltiplos. Superar as deficiências na capacitação técnico-científica, os vínculos precários do corpo profissional, as diferentes infraestruturas e capacidade técnica nas esferas subnacionais de gestão, o normativismo excessivo e os requisitos de harmonização de procedimentos se somam à manutenção de um distanciamento das políticas de saúde em geral, e das instâncias do controle social, do cidadão e do consumidor.

Quanto às limitações do estudo, embora a técnica de GF tenha sido conduzida por equipe experiente, com mobilização e estímulo à participação pelo moderador, é possível que as falas dos participantes como única evidência dos desafios à atuação da vigilância sanitária em serviços de saúde não expressem a totalidade da opinião dos participantes. Ressalta-se, ainda, que a composição intencional e de conveniência dos grupos pode refletir na capacidade de transferibilidade, ou seja, da possibilidade de que as conclusões sejam aplicadas a outros contextos ou para outros grupos.

\section{REFERÊNCIAS}

1. Costa EA. Regulação e vigilância sanitária: proteção e defesa da saúde. In: Rouquayrol MZ, Silva MGC, organizadores. Rouquayrol: epidemiologia \& saúde. 8a ed. Rio de Janeiro: Medbook; 2018. p. 461-86.

2. Pietro MSZ. Direito administrativo. 32a ed. Rio de Janeiro: Forense; 2019.

3. Freitas FP, Santos $B M O$. Irregularidades sanitárias como marcador de risco à saúde: um desafio para a vigilância sanitária. Vigil Sanit Debate. 2013;1(1):43-51. https://doi.org/10.3395/vd.v1n1.4

4. Morais LO, Friedrich K, Melchior SC, Silva MF, Gemal AL, Delgado IF. Eventos adversos e queixas técnicas relacionadas ao fio para sutura cirúrgica comercializada no Brasil. Vigil Sanit Debate. 2013;1(2):35-43. https://doi.org/10.3395/vd.v1n2.25

5. Costa EAM, Costa EA. Risco e segurança sanitária: análise do reprocessamento de produtos médicos em hospitais de Salvador, BA. Rev Saúde Pública. 2012;46(5):800-7. https://doi.org/10.1590/S0034-89102012000500006

6. Eduardo MBP, Novaes HMD. Análise de conformidade às normas técnicas de proteção radiológica dos serviços de radioterapia no Estado de São Paulo, Brasil. Cad Saúde Pública. 2004;20(2):256-67. https://doi.org/10.1590/S0102-311X2004000800020

7. Navarro MVT, Costa EA, Drexler GG. Radio diagnostic risk control: an approach of sanitary surveillance. Cienc Saúde Coletiva. 2010;15(Supl.3):3477-86. https://doi.org/10.1590/s1413-81232010000900022

8. Costa EAM, Santos QNF, Dantas IS. Risco em processamento de produtos para saúde em unidades básicas de Salvador, BA. Vigil Sanit Debate. 2018;6(2):38-43. https://doi.org/10.22239/2317-269x.00991
9. Durand C. A segurança sanitária num mundo global: os aspectos legais: o sistema de segurança sanitária na França. Rev Dir Sanit. 2001;2(1):60-78. https://doi.org/10.11606/issn.2316-9044.v2i1p59-78

10. Costa EAM. Vigilância sanitária em serviços de saúde: os desafios da prática. Vigil Sanit Debate. 2014;2(2):27-33. https://doi.org/10.3395/vd.v2n2.148

11. Silva JAA, Costa EA, Lucchese G. SUS 30 anos: vigilância sanitária. Cienc Saúde Coletiva. 2018;23(6):1953-62. https://doi.org/10.1590/1413-81232018236.04972018

12. Couto RC, Pedrosa TMG, Rosa MB. Erros acontecem: a força da transparência no enfrentamento dos eventos adversos assistenciais em pacientes hospitalizados. Belo Horizonte: Instituto Sobre Saúde Suplementar; 2016[acesso 19 out 2020]. Disponível em: https://www.iess.org. $\mathrm{br} /$ ? $\mathrm{p}=$ publicacoes\&id $=806$ \&id_tipo $=15$

13. Souza MKB, Lima YOR, Paz BMS, Costa EA, Santos R. Potencialidades da técnica de grupo focal para a pesquisa em vigilância sanitária e atenção primária à saúde. Rev Pesqui Qual. 2019;7(13):57-71. https://doi.org/10.33361/RPQ.2019.v.7.n.13.169

14. Laville C, Dionne J. A construção do saber: manual de metodologia em ciências humanas. Porto Alegre: Artmed; 1999.

15. Backes DS, Colomé JS, Erdmann RH, Lunardi VL. Grupo focal como técnica de coleta de dados e análise de dados em pesquisas qualitativas. Mundo Saúde. 2011;35(4):438-42.

16. Kinalski DDF, Paula CC, Padoin SMM, Neves ET, Kleinubing RE, Cortes LF. Grupo focal na pesquisa qualitativa: relato de experiência. Rev Bras Enferm. 2017;70(2):424-9. https://doi.org/10.1590/0034-7167-2016-0091 
17. Costa ICS, Souto AC. Indicadores em vigilância sanitária de alimentos. Rev Baiana Saúde Pública. 2011;35(4):826-44. https://doi.org/10.22278/2318-2660.2011.v35.n4.a257

18. Furtado JP, Campos GWS, Oda WY, Onocko-Campos R. Planejamento e avaliação em saúde: entre antagonismos e colaboração. Cad Saúde Pública. 2018;34(7):1-12. https://doi.org/10.1590/0102-311x00087917

19. Phelps C, Madhavan G, Rappuoli R, Levin S, Shortlife $E$, Colwell R. Strategic planning in population health and public health practice: a call to action for higher education. Milbank Q. 2016;94(1):109-25. https://doi.org/10.1111/1468-0009.12182

20. Maia C, Guilhem D. A regulação sanitária brasileira como parte da política de saúde: lacunas e desafios. Rev Panam Salud Publica. 2016;39(5):226-31.

21. Costa EA, Costa EAM. Controle sanitário do reuso de dispositivos médicos de uso único: um estudo de caso. Vigil Sanit Debate. 2020;8(2):106-12. https://doi.org/10.22239/2317-269x.01402

22. Agencia Nacional de Vigilância Sanitária - Anvisa. Ciclo de debates em vigilância sanitária: desafios e tendências: relatório do fórum nacional. Brasília: Agência Nacional de Vigilância Sanitária; 2015.

23. Costa $E A$, Souto $A C$. Área temática da vigilância sanitária. In: Paim JS, Almeida Filho N. Saúde coletiva: teoria e prática. Rio de Janeiro: Medbook; 2014. p. 327-41.

24. Covem EM. Descentralização das ações de vigilância sanitária. Cienc Saúde Coletiva. 2010;15(Supl.3):3318-28. https://doi.org/10.1590/S1413-81232010000900006

25. Patrício KP, Michaloski LCR, Rujula MJP. Vigilância em saúde. In: Ibañez N, Elia PEN, Seixas PHD, organizadores. Política e gestão pública em saúde. São Paulo: Hucitec; 2011. p. 354-73.
26. Alencar MLMS, Bacelar VMB, Magajewski F, Silva WM, Sousa AIA. Qualificação das ações de vigilância sanitária: harmonização e descentralização. Vigil Sanit Debate. 2019;7(4):111-18. https://doi.org/10.22239/2317-269x.01401

27. Paim JS, Teixeira CF. Configuração institucional e gestão do sistema único de saúde: problemas e desafios. Cienc Saúde Coletiva. 2007;12(Supl.):1819-29. https://doi.org/10.1590/S1413-81232007000700005

28. Machado MH, Ximenes Neto FRG. Gestão da educação e do trabalho em saúde no SUS: trinta anos de avanços e desafios. Cienc Saúde Coletiva. 2018;23(6):1971-9. https: / / doi.org/10.1590/1413-81232018236.06682018

29. Silva ACP, Pepe VLE. Vigilância sanitária: campo da promoção e proteção da saúde. In: Giovanella L, Escorel S, Lobato LVC, Noronha JC, Carvalho Al, organizadores. Políticas e sistema de saúde no Brasil. 2a ed. Rio de Janeiro: Fundação Oswaldo Cruz; 2012. p. 709 -38.

30. Oliveira AMC, Ianni AMZ. Caminhos para a vigilância sanitária: o desafio da fiscalização nos serviços de saúde. Vigil Sanit Debate. 2018;6(3):4-11. https://doi.org/10.22239/2317-269X.01114

31. Melo MAS, Bezerra JCB, Coleta MFD, Puente-Palacios KE, Coleta JAD, Bezerra ALQ. Comprometimento organizacional de trabalhadores da vigilância sanitária em municípios do estado de Goiás. Trab Educ Saúde. 2014;12(3):655-77. https://doi.org/10.1590/1981-7746-sip00018

32. Lucchese $G$. A vigilância sanitária no sistema único de saúde. In: Seta MH, Pepe VLE, Oliveira GO, organizadores. Gestão e vigilância sanitária: modos atuais do pensar e fazer. Rio de Janeiro: Fundação Oswaldo Cruz; 2006. p. 33-47.

\section{Contribuição dos Autores}

Lima YOR, Costa EA, Costa EAM, Araújo OS, Paz BMS, Souza MKB, Souza GS - Concepção, planejamento (desenho do estudo), análise, interpretação dos dados e redação do trabalho. Todas as autoras aprovaram a versão final do trabalho.

Os autores informam não haver qualquer potencial conflito de interesse com pares e instituições, políticos ou financeiros deste estudo.

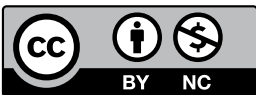

Licença CC BY-NC atribuição não comercial. Com essa licença é permitido acessar, baixar (download), copiar, imprimir, compartilhar, reutilizar e distribuir os artigos, desde que para uso não comercial e com a citação da fonte, conferindo os devidos créditos de autoria e menção à Visa em Debate. Nesses casos, nenhuma permissão é necessária por parte dos autores ou dos editores. 